ANDRIY TANASIYCHUK

Facoltà di Diritto Canonico San Pio X, Venezia

\title{
LE LETTERE DIMISSORIE NEL CODICE DEI CANONI DELLE CHIESE ORIENTALI
}

Sommario: Introduzione. - 1. Il ministro competente dell'ordinazione. 2. La persona competente che emana il documento. - 2.1. Vescovo eparchiale della stessa Chiesa sui iuris. - 2.2. Esarca patriarcale. - 2.3. Amministratore dell'eparchia. - 2.4. Il superiore del istituto di vita consacrata. - 2.5. Il Vescovo eparchiale di un'altra Chiesa sui iuris. -3 . Le conseguenze giuridiche dell'ordinazione. - Conclusione.

\section{Introduzione}

Lo scopo principale del presente studio riguarda alcune questioni, che pur non essendo di carattere pastorale, riguardano la vita della Chiesa. Si tratta di una serie di casi legati strettamente all'attività interna delle curie eparchiali e delle case principali degli istituti di vita consacrata. Uno di tali casi è il rilascio delle lettere (litterae dimissoriae) per l'ordinazione diaconale e presbiterale.

Il Codice dei Canoni delle Chiese Orientali (CCEO) ${ }^{1}$ nel canone 747, in cui si parla del modo di ricezione dell'ordinazione diaconale e presbiterale, stabilisce che il candidato può ricevere l'ordinazione in due modi: primo - essere ordinato dal proprio Vescovo eparchiale, secondo - essere ordinato da un Vescovo, che non è il proprio, che ha a disposizione i documenti richiesti dal diritto. Entrambi i modi richiedono diversi elementi che sono necessari affinché, per la Chiesa,

\footnotetext{
${ }^{1}$ Ioannis Pauli II, Codex Canonum Ecclesiarum Orientalium, 1990, Acta Apostolicae Sedis 82 (1990).
} 
l'atto giuridico sia lecito e produca quelle conseguenze giuridiche proprie dell'atto stesso favorendo il consolidamento della disciplina ecclesiale.

L'esame completo di questi elementi è l'oggetto di questo studio.

\section{Il ministro competente dell'ordinazione}

Riguardo la ricezione dell'ordinazione dal proprio Vescovo, occorre sottolineare che il diritto canonico orientale con il concetto di "Vescovo proprio" intende esclusivamente la persona che esercita l'ufficio del Vescovo eparchiale e non il Gerarca del luogo. Nel diritto canonico la nozione di Gerarca del luogo, oltre del Vescovo eparchiale, comprende anche il Protosincello e Sincello (cf. can. $984 \$ 2$ CCEO). Usando tale termine, ossia "Vescovo eparchiale", il Supremo Legislatore intende sottolineare il principale compito che gli è proprio e che si esprime nella responsabilità nei confronti del popolo di Dio a lui affidato e per il quale è stato designato. Perciò nel governo della propria eparchia gode di potestà ordinaria propria ${ }^{2}$. Queste caratteristiche tipiche dell'ufficio del Vescovo eparchiale lo distinguono dal Vescovo ausiliare e dal Vescovo coadiutore, indipendentemente dal fatto che lo sia stato o meno se designato come Protosincello o Sincello. Secondo il diritto questi ultimi non sono muniti della facoltà d'amministrare il sacramento dell'Ordine sui propri sudditi senza un debito permesso da parte del Vescovo eparchiale. In questo caso si parla di delega invece che di lettere dimissorie.

La norma del diritto presenta anche un altro modo con il quale un candidato può ricevere l'ordinazione e riguarda il diritto di un altro Vescovo a celebrare l'ordinazione diaconale o presbiterale. Sotto la nozione "altro Vescovo" la legislazione intende un Vescovo che non è il Gerarca del luogo per il candidato ${ }^{3}$. Inoltre la norma canonica

${ }^{2}$ Mentre per ciò che riguarda l'Esarca vedi A. TANAsIYCHUK, Esarca ed esarcato secondo il diritto canonico orientale. Alcune questioni di base e problematiche, Eastern Canon Law 4 (2014) (in stampa).

3 Sotto la nozione di Gerarca del luogo occorre intendere Vescovo eparchiale, Protosincello e Sincello: questi due ultimi uffici possono esercitare la funzione di 
richiede per questo atto giuridico un altro elemento importante: l'altro Vescovo, per poter ordinare lecitamente un candidato che non è suo proprio suddito, ha bisogno di ottenere la licenza dal Vescovo proprio del candidato. La dovuta licenza deve essere distinta dalla delega che di solito si concede al ministro che intende celebrare lecitamente i sacramenti per gli altri. Coloro che esercitano l'ufficio del Vescovo eparchiale tramite tale documento chiedono ad un altro Vescovo di ordinare il proprio candidato come diacono o presbitero. Questo documento, anche se viene considerato come un atto amministrativo, tuttavia non appartiene alla categoria dei decreti, rescritti o precetti, previsti nel canoni 1510-1539 del codice orientale.

Lo studio circa la necessità delle lettere dimissorie evidenzia anche un'altra questione importante, legata alla traduzione del codice orientale, per esempio in lingua ucraina. L'espressione litterae dimissoriae nella traduzione ucraina è stata tradotta con "lettere di dimissione". Nella lingua ucraina il temine "lettere di dimissione" relativo agli atti giuridici nella Chiesa è utilizzato anche per altre questioni. Questo termine infatti viene utilizzato nel caso in cui un ministro sacro esprime il desiderio di passare definitivamente dalla propria eparchia o esarcato sotto la giurisdizione di un altra circoscrizione ecclesiastica. Tale atto giuridico si presume valido nel caso in cui vi sia una licenza, nella forma scritta, di colui che esercita l'ufficio del Vescovo eparchiale (cf. can. 359 CCEO). Il problema ha origine nella traduzione in lingua ucraina dell'espressione litterae dimissoriae: il testo codiciale lo ha tradotto con "lettera di dimissione", invece che con "lettere dimissorie", che nello stesso codice si applica sia al passaggio definitivo di un ministro sacro ad un altra eparchia o esarcato che ai documenti necessari per poter essere ordinato da un altro Vescovo.

Quindi due atti giuridici nella Chiesa, cioè l'ordinazione sacra e il passaggio definitivo del chierico in un altra eparchia, che secondo il diritto canonico si distinguono tra di loro, nella traduzione ucraina del codice sono individuati usando lo stesso termine "lettera di dimissione", sebbene secondo il diritto canonico orientale questo termine

Vescovo ausiliare e di Vescovo coadiutore. 
identifichi solo un atto, cioè il passaggio definitivo del chierico all'altra eparchia o esarcato. Per trovare una soluzione in merito, prima che sia pronta la nuova redazione del CCEO in lingua ucraina, si propone che, per il rilascio dei documenti ecclesiastici necessari per compiere tali atti giuridici nella Chiesa, si usi il testo ufficiale, cioè lingua latina.

Inoltre si ritiene opportuno precisare un'altra questione legata al rilascio delle litterae dimissoriae. Tale documento è necessario ad un altro Vescovo come ministro dell'ordinazione o al candidato che riceve l'ordine. Infatti lo studio dei canoni mostra che questo documento è necessario soprattutto al ministro dell'ordinazione sacra per poter lecitamente (licite ministrant) ordinare un candidato che non è proprio suddito. Il codice prevede inoltre due condizioni principali perché il candidato riceva validamente l'ordinazione: deve essere di sesso maschile ed essere battezzato (cf. can. 754 del CCEO). L'elenco degli altri requisiti elencati nei canoni 758-761 della legislazione orientale riguardano solo la liceità del futuro atto giuridico nella Chiesa.

\section{La persona competente che emana il documento}

Le Litterae dimissoriae sono documenti che autorizzano un altro Vescovo ad amministrare l'ordinazione diaconale o presbiterale ad un candidato non proprio; vengono considerate come un elemento costitutivo del modo straordinario di ricevere l'ordinazione sacra; il modo ordinario che non richiede tali litterae può essere applicato solo a due condizioni: il ministro dell'ordinazione diaconale o presbiterale deve essere il Vescovo eparchiale proprio del candidato e sia il ministro che il candidato devono appartenere alla stessa Chiesa sui iuris.

Sottolineando la necessità che tale documento è diretto ad un altro Vescovo occorre passare allo studio della persona competente, secondo il diritto canonico orientale, che può rilasciare tale documento.

\subsection{Vescovo eparchiale della stessa Chiesa sui iuris}

Innanzitutto questa persona può essere lo stesso Vescovo eparchiale che esercita l'ufficio del Gerarca del luogo nei confronti del candidato all'ordinazione (cf. can. $748 \$ 3$ del CCEO). Una delle cause del rilascio di tale documento è il caso in cui il Vescovo, svolgendo 
l'ufficio del Vescovo eparchiale, nonostante appartenga alla stessa Chiesa sui iuris del candidato, ha la proibizione espressa dalla Sede Apostolica di ordinare diaconi o presbiteri i candidati sposati (cf. can. $758 \$ 3$ del CCEO). Altre situazioni in cui servono le litterae dimissoriae possono essere il caso in cui al Vescovo eparchiale non possa procedere all'ordinazione perché assente per malattia o a causa di un viaggio come un pellegrinaggio fuori dal paese. In questi casi l'ordinazione deve essere fatta da un altro Vescovo.

\subsection{Esarca patriarcale}

Altre persone che hanno il diritto di rilasciare le litterae dimissoriae ad un altro Vescovo, secondo il codice orientale, sono quelle strettamente legate all'esercizio dell'ufficio di Vescovo eparchiale, anche se esercitato per un tempo determinato. Possono essere, per esempio, l'amministratore eparchiale el'esarca patriarcale che secondo il diritto sono equiparati al Gerarca del luogo (cf. can. $984 \$ 2$ del CCEO), ma non sono Vescovi ordinati.

Nel caso in cui l'amministratore di una eparchia è un sacerdote, quindi è privo di diritto di amministrare il sacramento dell'ordine, per l'ufficio che svolge può chiedere ad un altro Vescovo (eparchiale o titolare) della stessa Chiesa sui iuris di ordinare il proprio candidato.

Invece quando si tratta dell'Esarca patriarcale, che è un sacerdote con potestà vicaria non propria ${ }^{4}$, benché abbia il diritto chiedere ad un altro Vescovo di ordinare il suo candidato, sarebbe meglio che tale richiesta fosse rivolta al Patriarca. Questa ultima modalità, cioè rivolgersi al Patriarca, trova il suo legittimo fondamento nel fatto che con tale richiesta si può concretizzare in due atti giuridici distinti compiuti dal Patriarca. Il primo riguarda l'ordinazione del candidato, invece il secondo è il permesso di poter ascrivere (incardinare) il neodiacono o neosacerdote nell'esarcato. Conferimento di tale permesso è stato previsto dal Supremo Legislatore (cf. can. 363, 1 CCEO).

${ }^{4}$ Cf. A. TAnasiychuk, Esarca ed esarcato secondo il diritto canonico orientale. Alcune questioni di base e problematiche, Eastern Canon Law 4 (2014) (in stampa) 
Il fatto che l'Esarca deve rivolgersi al proprio Patriarca si spiega non soltanto con il fatto che egli è un sacerdote, ma anche in relazione al tipo di potestà che ha ricevuto. Nel caso della potestà ordinaria, ma vicaria, l'Esarca patriarcale esercita il suo ufficio nel nome del Patriarca, che ordina il candidato e dà il permesso di ascriverlo nell'esarcato.

Invece nel caso in cui l'Esarca patriarcale è un Vescovo ordinato e svolge la potestà nel nome del Patriarca, secondo il nostro parere, la situazione è un po' diversa dalla precedente. Prima di ordinare il proprio candidato come diacono o presbitero, la cui conseguenza sarebbe l'ascrizione (incardinazione) all'esarcato, egli deve rivolgersi al Patriarca non solo per ottenere la licenza per l'ordinazione stessa, $\mathrm{ma}$ anche il permesso per l'ascrizione (incardinazione) del candidato all'esarcato (cf. can. 363, 1 CCEO).

\subsection{Amministratore dell'eparchia}

L'Amministratore dell'eparchia, indipendentemente dal fatto che sia Vescovo ordinato o meno, prima di incardinare all'eparchia un chierico o di dimetterlo dalla stessa, deve ottenere la licenza dal Patriarca (cf. can. 363, 1 CCEO). Invece, se questa struttura gerarchica si trova fuori del territorio canonico della Chiesa patriarcale, per poter ascrivere un chierico o dimetterlo dalla eparchia, l'Amministratore eparchiale può compiere questo atto giuridico solo dopo un anno dalla vacanza della sede eparchiale e col consenso del collegio dei consultori (cf. can. 363, 2 CCEO).

Anche in questo caso può succedere che l'Amministratore eparchiale non sia un Vescovo ordinato. Per poter compiere l'atto giuridico, cioè ascrivere (incardinare) un chierico all'eparchia tramite l'ordinazione diaconale, egli deve dare ad un altro Vescovo della stessa Chiesa sui iuris le litterae dimissoriae affinché possa ordinare il suo candidato. In questo caso per poter dare litterae dimissoriae l'Amministratore deve chiedere il parere del collegio dei consultori e ottenerne il consenso (cf. can. $750 \$ 1,2 \mathrm{CCEO).} \mathrm{Inoltre} \mathrm{questo} \mathrm{docu-}$ mento, secondo il nostro parere, dovrebbe essere annotato che tramite l'ordinazione diaconale il candidato viene ascritto (incardinato) alla 
struttura gerarchica di colui che ha rilasciato le litterae dimissoriae e non a quella in cui è stato ordinato.

Può anche capitare che l'Amministratore dell'eparchia sia un presbitero e nell'eparchia ci sia un Vescovo ausiliare. In questo caso sorge una domanda: quando l'Amministratore eparchiale si rivolge al Vescovo ausiliare perché sia lui ad ordinare il candidato della propria eparchia, deve concedere la litterae dimissoriae per l'ordinazione, oppure semplicemente un consenso verbale per il compimento dell'atto? Secondo le norme canoniche il Vescovo ausiliare deve ottenere la delega espressa e non le litterae dimissoriae dall'Amministratore dell'eparchia.

\subsection{Il superiore del istituto di vita consacrata}

La concessione delle litterae dimissoriae, può far pensare che esse vi siano solo nel rapporto tra la persona che esercita l'ufficio di Vescovo eparchiale e un altro Vescovo (eparchiale o titolare). Invece le norme canoniche che riguardano le litterae dimissoriae regolano anche la disciplina ecclesiastica di qualsiasi ministro dell'ordinazione che di candidato. Infatti le littarae dimissoriae sono usate dai superiori maggiori degli istituti di vita consacrata (cf. cann. 472, 532, 560 CCEO). Si tratta dei casi in cui il superiore maggiore del monastero, ordine o congregazione, che secondo il diritto canonico è il Gerarca per i suoi sudditi (cf. can. $984 \$ 3$ CCEO), si rivolge con tale letterae ad un Vescovo chiedendo l'ordinazione per un suo candidato. Solo nel caso in cui il superiore del monastero sui iuris eserciti anche l'ufficio di Esarca del monastero ed è Vescovo ordinato, si sostiene che goda personalmente del diritto di ordinare i propri sudditi.

\subsection{Il Vescovo eparchiale di un'altra Chiesa sui iuris}

La migrazione dei fedeli orientali cattolici nei nuovi territori di tradizione della Chiesa latina, dove non esistono strutture gerarchiche della propria Chiesa sui iuris, porta anche ad alcune conseguenze giuridiche. Tali fedeli possono ottenere un nuovo Gerarca del luogo 
(cf. can. 912, can. $916 \$ 5 \mathrm{CCEO}^{5}$ e un nuovo parroco (cf. can. 916 $\$ 4$ del CCEO). Il Vescovo diocesano o eparchiale di un'altra Chiesa sui iuris deve provvedere per una adeguata cura pastorale per questi fedeli (cf. can.193 CCEO) ${ }^{6}$ e per poter realizzare questo obbligo, dato dal Supremo Legislatore, sono necessari chierici oppure i candidati al sacerdozio che appartengono alla stessa Chiesa sui iuris dei fedeli. L'Ordinario/Gerarca del luogo, sforzando di organizzare la pastorale di questi fedeli, potrebbe, per esempio, invitare un certo gruppo dei seminaristi che fanno parte della Chiesa sui iuris di questi fedeli, dopo che questi hanno terminato la propria formazione in seminario, se sono in possesso dei requisiti prescritti dal diritto e dopo aver manifestato in modo scritto il desiderio di svolgere il servizio pastorale in questa diocesi, possono diventare ministri sacri (cf. can. $748 \$ 1$ CCEO). In tale situazione il Vescovo diocesano (eparchiale) per poter ordinare il candidato che continua ad appartenere ad una diversa Chiesa sui iuris, deve ricevere la debita licenza dalla Sede Apostolica (cf. can. $748 \$ 2$ CCEO e can. 1015 del CIC) ${ }^{7}$. In caso contrario sarà punito con le pene canoniche (cf. can. 1383 CIC e can. $1459 \$ 2$ CCEO).

Inoltre può avvenire un'altra situazione legata all'ordinazione del candidato che appartiene, per esempio, alla Chiesa Greco-Cattolica Ucraina, ma il suo domicilio si trova in Romania quindi il suo Vescovo eparchiale sarebbe il Vescovo della Chiesa Greco-Cattolica Romena. Qui occorre tenere presente un fatto particolare. Questi due soggetti (il ministro e il candidato) benché appartengono a due diverse Chiese sui iuris (Romena e Ucraina), hanno le loro radici ecclesiastiche nella stessa tradizione bizantina. Quindi si può sostenere che il Vescovo orientale rumeno può ordinare il proprio suddito che appartiene alla Chiesa Ucraina, anche senza la licenza della Sede Apostolica. Questo

\footnotetext{
${ }^{5}$ Cf. A. TAnasiychuк, Organizzazione della pastorale Greco-Cattolica Ucraina in Italia, in: Cristiani orientali e pastori latini, a cura di P. Gefaell, Giufferé Editore, Milano 2012, p. 465-477.

${ }^{6}$ Cf. Pontificio Consiglio della Pastorale per i Migranti e gli Itineranti, Istruzione Erga migrantes caritas Christi, 3 maggio 2004, in: Enchiridion Vaticanum 22/2418-2613, 1439-1511.

${ }^{7}$ Cf. Ioannis Pauli II, Codex Iuris Canonici, 1983, in AAS 75 II (1983), p. 1-317.
} 
orientamento proposto è sostenuto dall'analogia con la ricezione dell'indulto di biritualismo per un chierico orientale, documento che permette di celebrare i sacramenti in un altro rito. Osservando la pratica attuale della Congregazione per le Chiese Orientali, a cui spetta rilasciare tale atto amministrativo ${ }^{8}$, si può vedere che l'indulto di biritualismo si concede prevalentemente ad un chierico, quando vuole celebrare in altro rito, per esempio un sacerdote ucraino in rito latino, oppure in rito armeno e viceversa. Invece quando un chierico e i fedeli appartengono a diverse Chiese sui iuris ma con radici nella stessa tradizione ecclesiale per esempio bizantina (Chiesa ucraina, romena slovacca, greco-rutena) questo documento non è necessario.

\section{Le conseguenze giuridiche dell'ordinazione}

Esaminato il significato delle litterae dimissoriae e il loro valore per la vita della Chiesa, si propone un squadro relativo ad alcune conseguenze giuridiche che derivano dal conferimento dell'ordinazione sacra da un altro Vescovo.

Soprattutto occorre sottolineare che colui che riceve l'ordinazione, indipendentemente da chi lo ha ordinato, viene ascritto alla struttura ecclesiastica di colui che ha chiesto l'ordinazione sacra tramite le litterae dimissorie. Tra queste vi possono essere - l'eparchia, esarcato, istituto di vita consacrata.

Il conferimento dell'ordine sacro è legato ad un altro elemento, cioè al rito liturgico secondo il quale deve essere celebrato il sacramento. Questa particolarità si nota chiaramente nel canone 752 CCEO, nel quale si parla delle litterae dimissoriae che possono essere inviate dal Vescovo proprio ad un atro Vescovo della stessa Chiesa sui iuris e non a quella dell'ordinando. Così la norma canonica sottolinea il fatto che il ministro dell'ordinazione deve appartenere alla stessa Chiesa sui iuris a cui appartiene anche il candidato (cf. can. $748 \$ 2$ CCEO). Oltre questo stabilisce anche che il rito liturgico dell'ordinazione non deve essere distinto dal rito della Chiesa sui iuris, a cui appartengono il ministro e il candidato (cf. can. $674 \$ 2$ del CCEO).

${ }^{8}$ Cf. Giovanni Paolo II, Costituzione Apostolica Pastor Bonus, 58. 
Nella situazione contraria, cioè quando il ministro e il candidato appartengono a diverse Chiese sui iuris, il ministro, per poter amministrare il sacramento ha due possibilità: chiedere l'indulto di biritualismo e celebrare l'ordinazione secondo i libri liturgici della Chiesa sui iuris del candidato oppure ottenere la licenza dalla Sede Apostolica ed ordinare il candidato secondo i libri liturgici della propria Chiesa sui iuris e non del candidato (cf. can. $748 \$ 2$ CCEO).

Occorre precisare che l'ordinazione può essere celebrata in qualsiasi edificio cattolico orientale o latino, indipendentemente del luogo geografico della presenza reale del candidato e del ministro. Nel caso in cui l'ordinazione si celebra secondo il rito liturgico della Chiesa sui iris a cui appartiene sia il ministro che il candidato, per esempio, Chiesa ucraina, possono sorgere alcune domande legate ad una parte del rito durante il quale si pronuncia il giuramento che esprime il candidato prima dell'ordinazione presbiterale. Si presentano alcune soluzioni:

a) per esempio quanto si tratta di un diacono che già è ascritto (incardinato) alla struttura gerarchica di questa Chiesa (in Polonia, in Germania) indipendentemente da chi lo ordina, cioè da un altro Vescovo e quindi non suo proprio, il giuramento di obbedienza è fatta al Romano Pontefice, al Patriarca e Vescovo che ha conferito le litterae dimissoriae e loro successori;

b) mentre quando si tratta di un diacono che è ascritto (incardinato) alla struttura gerarchica di un altra Chiesa sui iuris, il candidato, anche se appartiene alla Chiesa ucraina, presta il giuramento di obbedienza esclusivamente al Romano Pontefice e al proprio Vescovo eparchiale o diocesano;

c) se l'esarca è un Vescovo ed esercita la potestà nel nome del Patriarca, ordina personalmente il proprio diacono. Il giuramento di obbedienza il candidato lo fa al Romano Pontefice, al Patriarca e all'Esarca. Qui viene citato l'Esarca perché governa una porzione del Popolo di Dio (cf. can. $311 \$ 1$ CCEO) che è stata a lui affidata ed anche perché, nella maggioranza dei casi in cui si esercita la potestà esecutiva, egli è equiparato al Vescovo eparchiale (cf. can. 313, can. 987 CCEO). 


\section{Conclusione}

Il diritto canonico dall'inizio del cristianesimo ha occupato un posto importante nella vita della Chiesa. I Concili ecumenici e sinodi locali, soprattutto nel primo millennio, hanno dedicato l'attenzione non solo alle questioni teologiche che sorgevano nelle comunità cristiane, ma anche ai problemi amministrativi e disciplinari. Basta solo analizzare le norme del concilio di Trullo che sottolineano l'obbligo della severa osservanza della disciplina canonica tra i cristiani ${ }^{9}$. Anche oggi la Chiesa svolgendo la missione della proclamazione del Vangelo nel mondo ha bisogno degli atti amministrativi che possano aiutarLa a svolgere la sua missione in modo più efficace.

Il tema delle litterae dimissoriae che sono oggetto di questo studio è una delle questioni che regolano i rapporti tra i Vescovi e tra i Vescovi e i presbiteri e i diaconi. L'esistenza di tale litterae nell'attività amministrativa della Chiesa permette di risolvere alcune delle situazioni relative a coloro che non hanno i requisiti per conferire l'ordine sacro.

Inoltre tali litterae, in modo particolare, sottolineano il fatto che ogni presbitero e diacono dopo la propria ordinazione non può avere una certa autonomia nella sua attività pastorale perché è parte costitutiva del presbiterio eparchiale, che sotto la direzione del proprio Vescovo eparchiale, realizza il fine pastorale della Chiesa.

\section{Dimissorial letters in the Code of Canons of the Eastern Churches}

The present study is dedicated to the administrative act of the competent person, who has the right to release dimissorial letters by exercising the proper governance in the Church. This document is necessary for the licit administration of the sacrament of Holy Orders. The eastern legislation that regulates the life of the Eastern Catholic Churches contains a vast number of such ecclesiastical subjects that are provided by the Supreme Legislator with the right to issue such a document. In addition, the Eastern canon law

${ }^{9}$ Cf. A. Tanasiychuk. Il Ius divinum nei canoni disciplinari del Concilio Trullano, in Ius divinum. Atti del XIII Congresso Internazionale di diritto Canonico (Venezia, 17-21 settembre 2008), a cura di J. I. Arrieta, Venezia 2010, p. 239-252. 
in its rules concerning the sacraments also show the liturgical nature of their celebration. Therefore it can be said that there is a strong and mutual relationship between the canonical norms and the liturgical rubrics among the Eastern Churches.

Parole chiave: Vescovo competente, Gerarca del luogo, sacramento degli ordini sacri, l'ordinazione, il ministro competente dell'ordinazione, lettere dimissorie, lettere di dimissione

KEY WORDS: the competent bishop, the hierarch of the place, the sacrament of Holy Orders, the ordination, the competent minister of ordination, dimissorial letters, dismissal letters

\section{BREVE NOTA SULL'AUTORE:}

ANDriy TANASIYchuK - prezbiter Eparchii Kołomyja-Czerniowce Ukraińskiego Kościoła Greckokatolickiego, wykładowca na Wydziale Prawa Kanonicznego San Pio X w Wenecji. 